\title{
Visually evoked potentials in eccentrically and centrally fixing amblyopes
}

\author{
RICHARD SREBRO \\ From the Department of Ophthalmology, Southwestern Medical School, 5323 Harry Hines Blvd, \\ Dallas, Texas 75235 , USA.
}

SUMMARY Visually evoked potentials to checkerboard pattern reversal were found to be nearly five times larger in eccentrically fixing amblyopic eyes than in centrally fixing amblyopic eyes when compared with the fellow non-amblyopic eye. The two groups of amblyopes had comparably poor visual acuity and differed in no other way save in their fixation behaviour. This suggests that at least two neurodevelopmental mechanisms subserve human amblyopia and that only one of these resembles the animal model of visual deprivation.

It has been proposed that monocular visual deprivation in animals may serve as a model for human amblyopia.' If this hypothesis is correct, then the pathophysiology of human amblyopia is subserved by a reduction of neural input from the amblyopic eye to the visual cortex. In humans visually evoked potentials (VEPs) reflect the amount of neural input to the visual cortex. Several studies have shown that the VEPs evoked by contrast reversal of checkerboard or grating patterns are often smaller when the amblyopic eye is stimulated than when the fellow non-amblyopic eye is stimulted." "Comparisons between the VEPs due to stimulations of the amblyopic and fellow non-amblyopic eye of the same subject are useful because, while VEP amplitudes vary widely from subject to subject, they are more nearly equal for stimulation of the two eyes of the same normal subject. However, these studies have failed to provide evidence of a relationship between VEP amplitude and visual acuity. Since the hypothesis that human amblyopia represents a reduction in neural input to the visual cortex implies such a relationship, it is of some importance to determine if amblyopes with comparable visual acuities have comparable VEP amplitudes. The study reported here shows that VEP amplitudes are substantially smaller in centrally fixing amblyopic eyes than in eccentrically fixing amblyopic eyes with comparable visual acuities. This result challenges the previously proposed monolithic hypothesis for the pathophysiological mechanism of human amblyopia and raises

Correspondence to R. Srebro, MD. the possibility of a functionally distinct neurodevelopmental process that leads to eccentric fixation.

\section{Material and methods}

VEPs were evoked by high-contrast black and white checkerboard pattern reversal stimuli generated on a video display. The stimulus subtended approximately 12 arc degrees (diagonal of video screen), contained 40 arc minute checks, and had a space-averaged luminance of approximately 10 foot-lamberts ( 34 $\left.\mathrm{cd} / \mathrm{m}^{2}\right)$. Subjects viewed the display monocularly with a natural pupil using their optimal refractive corrections. A small fixation cross was provided. The electrode placement followed the 10-20 International Electrode Placement System with the positive electrode at $\mathrm{Oz}$, the negative electrode at $\mathrm{Cz}$ and ground at $\mathrm{Pz}$. Each VEP was the average response to 256 pattern reversals at $1.88 \mathrm{~Hz}$. The bandpass of the recording system was 1 to $100 \mathrm{~Hz}$ (3 decibel points). The amblyopic and fellow non-amblyopic eye of each subject was tested in the same recording session. Repeated measurements often widely separated in time (months) revealed no substantial differences in either VEP waveform or amplitude in several subjects.

The data presented here derive from 10 amblyopic and 16 normal subjects. All were adults. All were screened by a complete eye examination, including a cycloplegic and manifest refraction and visuscopy measurements of fixation. The amblyopic subjects studied were selected from 51 amblyopes who volunteered on the basis of the visual acuity of the 
amblyopic eye (20/100 or worse), and fixation (either unequivocally stable and central, or unequivocally stable and eccentric). The eccentrically fixing amblyopes fixated with retinal points ranging from 2 to 4 arc degrees from the centre of the fovea. The non-amblyopic eye was required to be entirely normal and to have visual acuity $20 / 20$ or better. These criteria for inclusion in the study were adopted a priori.

\section{Results}

Fig. 1 shows VEPs for both the nonamblyopic eyes and the amblyopic eyes of 2 amblyopic subjects. The two VEPs at the top of the figure, marked $E$, are those of an eccentrically fixing amblyope (subject 12 , Table 1). The two VEPs at the bottom of the figure, marked $\mathrm{C}$, are those of a centrally fixing amblyope (subject 10, Table 1). The topmost VEP of each pair is that of the non-amblyopic eye. Although the visual acuity of the eccentrically fixing amblyopic eye $(20 / 400)$ was worse than that of the centrally fixing amblyopic eye $(20 / 200)$, the VEP due to stimulation of the eccentrically fixing amblyopic eye is nearly equal in amplitude and waveform to that of the fellow non-amblyopic eye. But the VEP due to stimulation of the centrally fixing amblyopic eye is very much smaller than that of the fellow non-amblyopic eye.

Table 1 lists the ratios of VEP amplitude due to stimulation of the amblyopic eye to that due to stimulation of the fellow non-amblyopic eye for each amblyopic subject studied. The amplitude measure-
Table 1 Clinical data and VEP ratios for amblyopic subjects

\begin{tabular}{|c|c|c|c|c|}
\hline $\begin{array}{l}\text { Subject } \\
\text { number }\end{array}$ & $\begin{array}{l}\text { Type of } \\
\text { amblyopia }\end{array}$ & $\begin{array}{l}\text { *Visual } \\
\text { acuity }\end{array}$ & $\dagger$ Fixation & $\begin{array}{l}\ddagger V E P \\
\text { amplitude } \\
\text { ratio }\end{array}$ \\
\hline 10) & Strabismic & $20 / 2(0)$ & Central & 0.36 \\
\hline 11 & Anisometropic & $20 / 200$ & Central & 0.08 \\
\hline 34 & Strabismic & $20 / 10(0)$ & Central & 0.18 \\
\hline 194 & Strabismic & $2(0 / 2(x)$ & Central & $0 \cdot 14$ \\
\hline 164 & Strabismic & $20 / 2(x)$ & Central & $0 \cdot 10$ \\
\hline 117 & Strabismic & $20 / 20(0)$ & Eccentric & 0.52 \\
\hline 12 & Strabismic & $2(0 / 4())$ & Eccentric & 0.84 \\
\hline 33 & Anisometropic & $2(0 / 2(x)$ & Eccentric & 0.93 \\
\hline 141 & Strabismic & $20 / 4(x)$ & Eccentric & 0.55 \\
\hline 134 & Strabismic & $20 / 100$ & Eccentric & 0.96 \\
\hline
\end{tabular}

*Snellen line.

+Visuscopy.

$\ddagger$ Amplitude VEP amblyopic cye/fellow non-amblyopic eyc.

ment itself is shown in Fig. 1, topmost trace. For the 5 centrally fixing amblyopic subjects this ratio ranged from $0 \cdot 10$ to $0 \cdot 36$, while for the five eccentrically fixing amblyopic subjects it ranged from 0.52 to 0.93 . The average value of the VEP amplitude ratio ( \pm standard error) was $0 \cdot 17( \pm 0 \cdot 12)$ for the centrally fixing amblyopic subjects, and $0.76( \pm 0 \cdot 21)$ for the eccentrically fixing amblyopic subjects. This difference of means in significant at less than the 0.005 level (Student's $t=4 \cdot 89$, degrees of freedom $=8$ ).

The average VEP amplitude ratio ( \pm standard error) was $(0.95( \pm 0 \cdot 18)$ for the 16 normal control subjects (lesser/greater). The difference of means

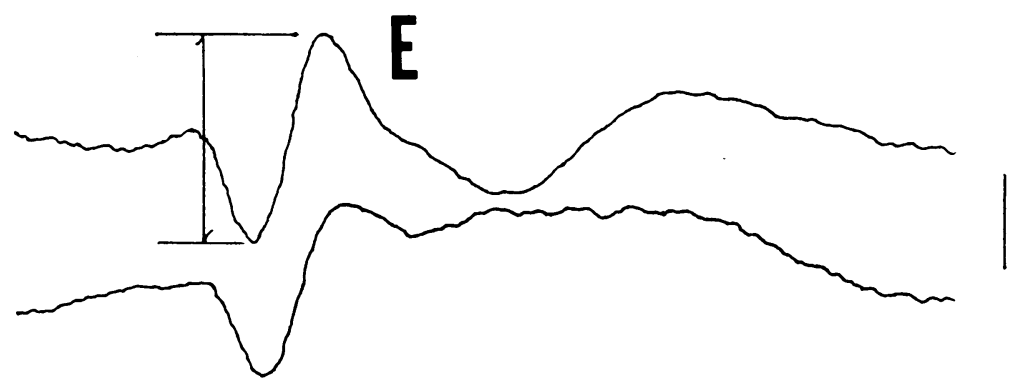

Fig. 1 Visually evoked potentials for two amblyopic subjects. Each trace shows 300 ms following checkerboard pattern reversal. See under 'Material and methods' for details of measurement. $\mathrm{E}=$ VEPs for subject I2 (see Table I), an eccentrically fixing amblyope with visual acuity 20/400. $\mathrm{C}=$ VEPs for subject 10, a centrally fixing

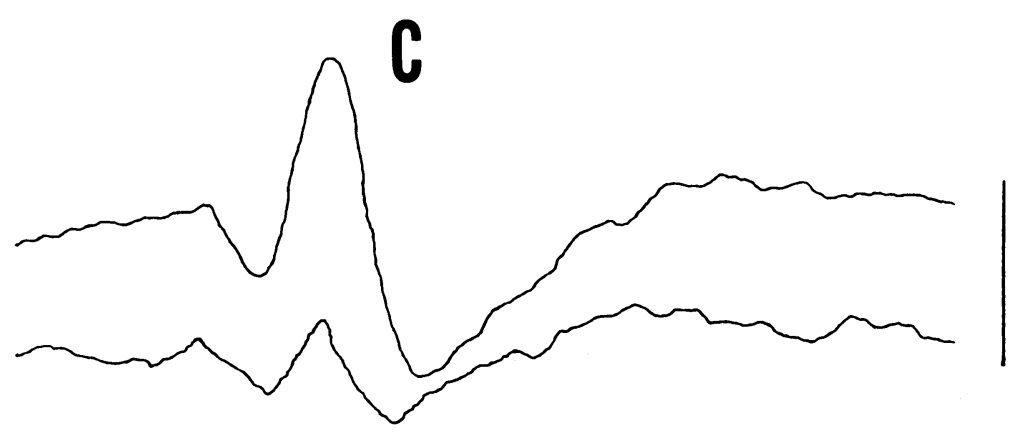
amblyope with visual acuity 20/200. The top trace in each pair is due to stimulation of the non-amblyopic eye. The bottom trace of each pair is due to stimulation of the amblyopic eye. Calibration mark equals 4 microvolts. Top trace of Figure illustrates VEP amplitude measurement. 
between the centrally fixing amblyopes and the normal controls was -0.78 and is significant at less than the $0 \cdot 005$ level (Student's $t=8 \cdot 64$, degrees of freedom $=19$ ). The difference of means between the eccentrically fixing amblyopes and the normal controls was -0.19 and is questionably significant at the $0 \cdot 05$ level (Student's $t=1 \cdot 88$, degrees of freedom $=19$ ).

\section{Discussion}

The criteria restricting the entry of amblyopic subjects into the study reported here resulted in a study group of 10 amblyopic subjects drawn for 51 amblyopic subjects who were screened. There were several objectives that led to the adoption of such restrictive criteria. First, a group of amblyopes with relatively homogeneous visual acuities were selected so that it would be reasonable to compare them without having to deal with the intervening variable of visual acuity, which can obscure results. Secondly, amblyopic subjects with relatively poor visual acuity in the amblyopic eye were selected because, with the visuscopy, it is frequently difficult to be sure if fixation is central or eccentric if the amblyopic eye has visual acuity much better than 20/100. The resolution of visuscopy, not better than 1 arc degree ${ }^{7 x}$ is too poor reliably to detect the small degrees of eccentric fixation that may exist in amblyopic eyes with relatively good visual acuity. Finally, the fixation had to be unequivocally stable and categoriseable so that there was no doubt whatever about it in any case. Therefore, although small, the select group of amblyopic subjects was well constituted to compare unambiguously the VEP amplitudes in two groups of amblyopes with comparable visual acuities. The use of eccentric fixation as a possible discriminant was based mostly on intuition.

All VEP amplitudes are presented here as fractional values, comparing the VEPs due to stimulation of the two eyes of the same subject. There are several reasons why VEP amplitude varies from subject to subject. The thickness of the scalp and skull varies among subjects and affects the VEP amplitude." "I The topography of the cortical representation of the visual field varies among subjects, $" 12$ so that for a fixed electrode placement VEP amplitude also varies. However, these factors do not affect comparative VEP measures due to stimulation of the two eyes of the same subject. The 16 normal subjects studied here had VEPs whose amplitudes were generally within $20 \%$ of each other for stimulation of the two eyes. The stimulus for the VEP contained 40 arc minute checks. This check size was selected because stimuli containing much smaller checks cause VEPs whose amplitudes are prone to wider variation from measurement to measurement in the same subject, and because stimuli containing much larger checks are insensitive to the amblyopic defect.

The results show unequivocally that eccentrically fixing amblyopic eyes have VEP amplitudes that are nearly five times larger than those of centrally fixing amblyopic eyes when compared with the fellow nonamblyopic eye. This difference is statistically significant in spite of the small sample size. As seen in Table 1 , there is virtually no overlap between the two groups in this regard. This difference in VEP amplitude cannot be attributed to differences in visual acuity between the two groups, because, as Table 1 shows, there was little difference in this regard. Since most of the amblyopic subjects were strabismic except for one anisometropic amblyope in each group, the difference in VEP amplitude cannot be attributed to the type of amblyopia, and the result may hold for both major types of amblyopia. The difference in VEP amplitude between centrally and eccentrically fixing amblyopic eyes cannot be ascribed to differences in the position of the checkerboard stimulus display in the visual field of the two classes of subjects, because the size of the display was much larger than the magnitude of the eccentric fixation in any subject (4 arc degrees or less).

The pathophysiology of eccentric fixation is only poorly understood. ${ }^{8.3} 17$ Two different hypotheses have been proposed to account for it. One hypothesis suggests that, when viewing a small target monocularly with the amblyopic eye, an eccentric fixator rotates his eye so that the image of the target falls on the retinal locus subserving the best attainable resolution - that is, near the edge of the amblyopic relative central scotoma. The other hypothesis suggests that eccentric fixation is the result of faulty relative spatial localisation-that is, an anomalous principal visual direction associated with the eccentric fixation point rather than the centre of the fovea. Neither hypothesis offers an entirely satisfactory explanation for the existence of eccentric fixation. For example, the first hypothesis does not explain why a particular retinal point along the perimeter of the central scotoma becomes the fixation point. And the second hypothesis is mute on the issue of whether eccentric fixation is the cause of the reduced visual acuity. If the amblyopic relative central scotoma is a psychophysical manifestation of reduced neural input to the visual cortex, then the results presented here suggest that the scotoma is considerably smaller in eccentrically fixing amblyopic eyes than it is in centrally fixing amblyopic eyes. Indeed, the results shown in Table 1 suggest that at least some eccentrically fixing amblyopic eyes with poor visual acuity have VEPs whose amplitudes are negligibly smaller than those of their fellow non-amblyopic counterparts, 
and thus may have little or no relative central scotomata at all. Thus the findings may support the hypothesis that eccentric fixation results from a primary mechanism involving altered spatial localisation and that it may be wholly or partly responsible for the loss of visual acuity.

The findings do not support the hypothesis that a monolithic pathophysiological mechanism subserves human amblyopia, namely a reduction in the neural input from the amblyopic eye to the cortex. The brain may have at least two neurodevelopmental mechanisms with which to cope with failure of binocular single vision and the conflict of information provided by the two eyes that this failure implies. One mechanism may involve partial elimination of the input from one eye and is thus similar in effect to experimental visual deprivation. The other may involve a remapping of relative visual direction from one eye so as to allow the formation of cyclopean perceptions that are compatible with real objects. This mechanism has no known experimental counterpart. Both mechanisms may be used to a different extent in each individual case. Although speculative, this hypothesis has testable predictions. First, at least some eccentrically fixing amblyopic eyes will be found to have normal visual acuity at the fovea; these eyes will have VEPs that are indistinguishable from those of their fellow non-amblyopic eyes. Secondly, in amblyopes young enough to respond to patching, the results of this treatment will bear some relationship to their VEPs recorded before treatment begins. Those amblyopes, for whom there is a considerable difference between the VEPs of the two eyes, will respond to patching with a steady improvement in visual acuity through its course. Also the VEP of the amblyopic eye will show a steady increase in amplitude. This prediction can be made because, by hypothesis, the pathophysiological mechanism of the amblyopia in this group is in effect similar to that of visual deprivation in animals, where reverse deprivation has predictable results. However, for those amblyopes whose VEPs are nearly equal in amplitude in the two eyes, the results of patching are unpredictable, because they do not fit any known experimental model and because it is not clear if patching alone can provoke a reversal of eccentric fixation back to central fixation. Thus these amblyopes may either not respond to patching at all, or if they do respond the improvement in visual acuity will be abrupt, and in any event the VEP of the amblyopic eye will not change.

\section{References}

I Ikeda $\mathrm{H}$, Wright MJ. A possible ncurophysiological basis for amblyopia. Br Orthopt J 1975; 32: 2-13.

2 Lombroso C. Duffy F, Robb R. Sclective suppression of cercbral evoked potentials to patterned light in amblyopia ex anopsia. Electroencephalogr Clin Neurophysiol 1969; 27: 238-47.

3 Sokol S, Bloom B. Visually evoked cortical responses of amblyopes to a spatially alternating stimulus. Invest Ophthalmol Visual Sci 1973; 12: 936-9.

4 Arden GB, Barnard WM, Mushin AS. Visually evoked responses in amblyopia. BrJ Ophthalmol 1974; 58: 183-92.

5 Vinon U, Jakobovitz L. Aucrbach E. The visually evoked response to stationary checkerboard patterns in children with strabismic amblyopia. Invest Ophthalmol Visual Sci 1974; 13: 293-6.

6 Levi DM, Harwerth RS. A sensory mechanism for amblyopia: clectrophysiological studics. Am J Optom Physiol Opt 1978; 55: 163-71.

7 Lawwill T. Fixation pattern of the light and dark adapted eye. Ain J Ophthalmol 1966; 61: 1416-9.

8 Van Noorden GJ. Pathogenesis of ecentric fixation. Doc Ophthalmol 1967: 23: 263-94.

9 Rush S, Driscoll DA. EEG clectrode sensitivity-an application of reciprocity. IEEE Trans Biomed Eng 1969; BME 16: 15-22.

10) Kavanagh RN, Darcey TM, Lehman D, Fender DH. Evaluation of methods for threc-dimensional localization of electrical sources in the brain. IEEE Trans Biomed Eng 1978: BME 25: 421-9.

11 Brindley GS. Lewin WS. The sensations produced by electrical stimulation of the visual cortcx. J Physiol (Lond) 1968; 196: 479-93.

12 Brindlcy GS. The variability of the human striate cortex. J Physiol (Lond) 1972; 255: 1-3P.

13 Flom MC, Wcymouth FW. Centricity of Maxwell's spot in strabismus and amblyopia. Arch Ophthalmol 1961; 66: 136-44.

14 Von Noorden GK. Mackensen G. Phenomenology of ecentric fixation. Ain J Ophthalinol 1962; 53: 642-60).

15 Von Noorden GK. The etiology and pathogenesis of fixation anomalics in strabismus. Trans Am Ophthalmol Soc 1969; 67: 698-751.

16 Hess RF. On the relationship between strabismic amblyopia and eccentric fixation. BrJ Ophthalmol 1977; 61: 767-73.

17 Kirschen DG, Flom MD. Visual acuity at different retinal loci of cccentrically fixating functional amblyopes. Am J Optom 1978: 55: $144-5()$. 